A. Pramesh Rao, G. Swarup and Gopal-Krishna, eds.

\title{
Practical Lessons from Low Frequency Imaging with the VLA
}

\author{
Christina K. Lacey and Namir E. Kassim \\ NRL-Code 7213, Washington DC, 20375, USA
}

\begin{abstract}
.
Many lessons gained in imaging 74 and $330 \mathrm{MHz}$ VLA data are of practical interest to future users of the GMRT and other low frequency observers. Three important issues are 1) radio frequency interference excision, 2) self-calibration, and 3) wide-field imaging. Recently developed tasks to address these and other problems encountered in low frequency VLA data reduction now exist or are being developed in both AIPS and AIPS++. We will describe how we use some of these new tools to routinely generate wide-field, low frequency images with sensitivities approaching thermal or classical confusion limits.
\end{abstract}

\section{Introduction}

The $74 \mathrm{MHz}$ system at the $\mathrm{VLA}^{1}$ is described in detail by Perley et al. (in these proceedings). This paper reviews procedures that have been developed in the Astronomical Imaging Processing System (AIPS) to efficiently calibrate and image low frequency data. For full documentation and step-by-step instructions of the calibration and imaging of low frequency observations at the VLA see Kassim et al. (1999).

Issues key to low frequency imaging begin with gain and phase calibration, which are initially set by observing a single strong source, and only later fine tuned by self-calibration. Another issue is radio frequency interference (RFI), which for the VLA environment required the development of a specialized task to properly remove contaminated data. To do this, observations are now routinely conducted in spectral line mode, which also alleviates the effects of bandwidth smearing for sources far from the phase center. Finally, self-calibration and non-coplanar baseline wide field imaging algorithms must be run in tandem to properly deconvolve the myriad of background sources, which normally are detected in the resulting large angular scale images.

\footnotetext{
${ }^{1}$ The National Radio Astronomy Observatory is a facility of the National Science Foundation operated under cooperative agreement by Associated Universities, Inc.
} 


\section{Initial Calibration and RFI Excision}

Cygnus A, the second strongest source (after Cas A) at $74 \mathrm{MHz}$ at approximately $16,000 \mathrm{Jy}$, is the main calibrator for low frequency observations. Other strong sources can be used if necessary, a list of suitable calibrators and models is being compiled (Kassim 1999). If the source is resolved, as in the case of Cygnus A, a good model is also required. The traditional method of using a nearby phase calibrator that is observed frequently throughout the observation is not used. Cygnus A sets the gains which do not vary appreciably for days or even weeks at a time, and the phases transferred from Cygnus are good enough for the shorter spacings $(<10 \mathrm{~km})$ to retain sufficient coherence to generate an initial, low resolution model of the target field. Self calibration then does the rest, driven by the large amount of real signal (typically $>20 \mathrm{Jy}$ at $330 \mathrm{MHz},>100$ Jy at $74 \mathrm{MHz}$ ) normally present within the large field of view.

The basic procedure begins with the AIPS routines BPASS and CALIB, which are run on Cygnus A to set the bandpass shape, antenna gains, and initial phases. The key to using such a bright source is that the signal to noise is sufficient on individual channels so that the resulting solutions are relatively unaffected by RFI, which can therefore be ignored at this stage. After the antenna gains and phases are transferred from Cygnus A to the target source, the latter is examined for RFI which can then be excised using the new AIPS routine FLGIT described below.

The low frequency RFI at the VLA is generally narrow band, internally generated at $74 \mathrm{MHz}$ and mainly externally generated at $330 \mathrm{MHz}$ (see Perley et al. in these proceedings). (To date not a single case of externally generated RFI has been seen at $74 \mathrm{MHz}$.) The RFI has a strong baseline dependence, being much worse on the short spacings and hence a bigger problem for the more compact configurations. Thus, A array is always the cleanest, while data from $D$ array is always the most affected.

The standard procedure is to use the AIPS task SPFLG, which allows one to visually examine the amplitudes (or phases) of the visibility data as a function of spectral line channel and time. To eliminate the time-consuming effort of examining all the individual baselines, SPFLG is run on a few representative baselines, including at least one short, intermediate, and long spacing. These SPFLG sessions are used to determine the thermal noise levels in RFI-free regions and to define a clean mask of mainly RFI-free channel ranges. The new AIPS task FLGIT, developed by E. Greisen, then uses these parameters to excise the RFI from the cross power spectra on a channel by channel basis, taking into account the bandpass shape. A few baselines of the spectral data set that come out of FLGIT can then be examined using SPFLG to determined if the FLGIT parameters were set properly.

\section{Self-Calibration and Wide-Field Imaging}

Self-calibration and imaging are the last stages in the $74 / 330 \mathrm{MHz}$ imaging process. Initially, it was thought that phase transfer from $330 \mathrm{MHz}$ observations would be required to generate an ionospheric model to "unwind" the effects of the ionosphere on the $74 \mathrm{MHz}$ phases (Kassim et al. 1993). However, in 
practice there almost always appears to be sufficient coherence on real signal on the shorter spacings as defined from the transfered Cygnus phases, so that straight forward self-calibration can proceed at $74 \mathrm{MHz}$ without an additional ionospheric model. There are some exceptions to this, such as the Galactic center (see paper in these proceedings by Anantharamaiah et al.) Phase self-calibration is typically run on times scales of a few minutes. Amplitude self-calibration is usually run on scan lengths (typically $5-10$ minutes) at $330 \mathrm{MHz}$ but is almost never used at $74 \mathrm{MHz}$, where little improvement over the gains set by Cygnus are required.

To achieve thermal or classical confusion limited imaging, deconvolution must take into account the non-coplanar baseline effects at the VLA. Successful wide-field imaging algorithms have existed for some time outside of AIPS (see e.g. Larosa et al. 2000 for an example of their application in SDE), but the AIPS task IMAGR can perform this now. The 3-D imaging is accomplished by utilizing a polyhedron algorithm which breaks the large field of view into many sub-images or facets, each of which subtends a small enough field of view that it can be approximated by a two dimensional plane. The different position angles and tangent lines to each field are calculated separately for each field.

The 3D IMAGR is driven by the new AIPS task SETFC, first developed by T. J. W. Lazio at NRL and then refined by B. Butler and E. Greisen at NRAO. SETFC calculates the phase center and size of the facets, forming a central overlapping group of fields referred to as a fly's eye. SETFC also generates outlying facets for strong confusing sources that are located further out in the primary beam. This targeted faceting approach precludes the computational expense of having to pixellate the entire primary beam. SETFC uses the NVSS catalog to set the outlier fields, since in almost all cases strong confusing sources at 74 and $330 \mathrm{MHz}$ are also prominent NVSS sources.

IMAGR and CALIB are then run iteratively until no noticeable improvement in image fidelity is achieved. At this stage the AIPS task FLATN is used to mosaic together the central set of overlapping facets forming the fly's eye and thereby creating the final desired image of the target field.

\section{W49B}

As an example of $74 \mathrm{MHz}$ data reduction and imaging, an image of $\mathrm{W} 49 \mathrm{~B}$ is presented. W49B was observed on 29 June 1999 at the VLA in A configuration. SETFC generated a central $1^{\circ} \mathrm{fly}$ 's eye and used the NVSS catalog to determine a list of outlying source fields within $30^{\circ}$ of the phase center. This limited the outlying fields to a manageable number. Cygnus $\mathrm{A}$ was the calibrator, and loops of CALIB and IMAGR were then run sequentially as described above to fine tune the phases and generate a final image. The $74 \mathrm{MHz}$ image of $\mathrm{W} 49 \mathrm{~B}$ is presented in Figure 1 along with a $330 \mathrm{MHz}$ image. Although the angular resolution of the $330 \mathrm{MHz}$ is almost three times greater than that of $74 \mathrm{MHz}$, it is clear that there is a definite difference in the appearance of the remnant at $74 \mathrm{MHz}$ compared to $330 \mathrm{MHz}$. The emission on the right (southwest) side of the remnant is suppressed compared to the rest of the remnant. This is likely due to thermal ISM absorption along the line of sight. Many such cases of freefree absorption towards Galactic SNRs at low frequency have been inferred from 

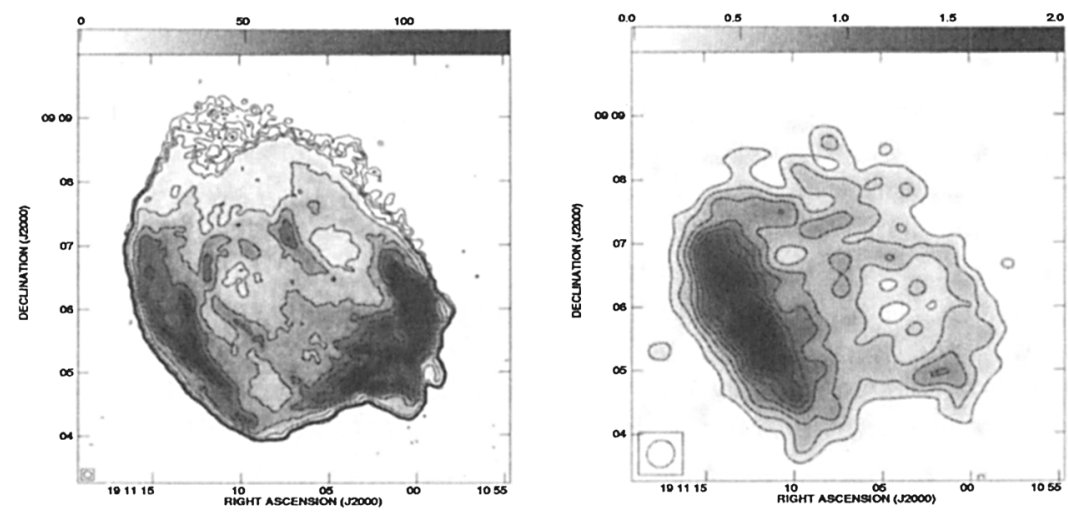

Figure 1. W49B images: $330 \mathrm{MHz}$ on left and $74 \mathrm{MHz}$ on right.

integrated spectral turnovers (e.g. see Kassim 1989) but to our knowledge this is the first case in which this absorption has been spatially resolved.

\section{Conclusion}

The $74 \mathrm{MHz}$ and $330 \mathrm{MHz}$ systems at the VLA have inspired the development of a suite of data reduction software and reduction procedures specifically catering to the handling of special problems encountered at long wavelengths. These include RFI excision and wide field imaging software packages now within AIPS. The $74 \mathrm{MHz}$ system in particular has inspired a number of new and creative solutions which are also now being extended into AIPS++. As more data is obtained, these techniques continue to be refined, generating lessons which should prove extremely useful to future users of the VLA, GMRT, and proposed future low frequency facilities with even greater imaging power at the longest wavelengths $(<150 \mathrm{MHz}$ ) such as the Low Frequency Array (LOFAR, see paper by Kassim et al. in these proceedings.)

Basic research in radio astronomy at the Naval Research Laboratory is supported by the Office of Naval Research.

\section{References}

Anantharamaiah et al., in these proceedings

Kassim, N. E., Lazio, T. J. W., Erickson, W. C. Crane, P. C., Perley, R. A., \& Hicks, B., in these proceedings

Kassim, N. E., Perley, R. A., \& Lazio, T. J. W. 1999, "Low-Frequency Data Reduction at the VLA", http://rsd-www.nrl.navy.mil/7213/lazio/tutorial/

Kassim, N. E., Perley, R. A., Erickson, W. C. \& Dwarakanath, K. S. 1993, AJ, 106,2218

Kassim, N. E. 1989, ApJS, 71, 799

LaRosa, T., Kassim, N. E., Lazio, T. J. W., \& Hyman, S. D. 2000, AJ, 119, 207

Perley, R. A., Erickson, W. C., \& Kassim, N. E., in these proceedings 